\title{
Custom and standard care: implications for the future role of doctors in mental health
}

\author{
Riadh T. Abed ${ }^{1}$
}

The Psychiatrist (2010), 34, 505-506, doi: 10.1192/pb.bp.110.031658

${ }^{1}$ Rotherham Doncaster and South Humber Mental Health NHS Foundation Trust, St Catherine's Hospital, Doncaster

Correspondence to Riadh T. Abed (riadh.abed@rdash.nhs.uk)
Summary Bohmer's concepts of custom and standard care are briefly discussed with specific reference to their implications for the role of doctors in mental health services. It is argued that these concepts help to clarify the unique contribution of doctors within multidisciplinary teams and help define the limits of standardisation in mental health services.

Declaration of interest None.
The concept of multidisciplinary team working has been evolving over the past three decades and has recently been augmented by the New Ways of Working initiative. ${ }^{1,2}$ This has attempted to define the contribution that doctors make to the working of multidisciplinary teams in mental health services and also to develop greater clarity regarding the role and contribution of other disciplines. New Ways of Working attempts to provide a model for a rational division of labour that would form the basis of a well-functioning modern mental health team. This would involve a move away from solitary and unsupervised practice to more teambased, scrutinised clinical decision-making.

Although the psychiatrist within this model is no longer the clinician who carries the ultimate clinical responsibility for all the team's workload, he or she will continue to have a pivotal consultative role within the team. The consultant will be involved with the most complex and demanding cases and should relinquish routine clinical work.

However, although the principles of New Ways of Working are undoubtedly sound, there remains a degree of uncertainty as to how these principles can be applied in practice. Some of the difficulty relates to the assumption that there is a clear understanding of the nature of the contribution of mental health practitioners to the process of mental healthcare. Also, it is assumed that patients' journeys and pathways of care are well mapped out, with a clear understanding of the value added by each step or intervention. I suggest that much more needs to be done to clarify the nature of value-adding clinical activity in mental health as this will provide the basis for a more rational and evidence-based design of care systems that makes best use of the skills and competencies of all disciplines.

\section{Bohmer's concept}

With this in mind, I suggest that Bohmer's concepts of standard versus custom care provides a useful framework to take this analysis a few steps further. ${ }^{3}$ Bohmer proposes that healthcare involves two distinct but, at times, interrelated activities; standard care, which involves dealing with wellunderstood clinical problems that have clear evidence-based solutions, and custom care, which deals with problems that are not well understood, are atypical or entirely novel.

Standard care involves the application of clear and well-understood interventions that include investigations and treatment through a sequential process. High-quality standard care will involve a high degree of adherence to known and well-understood good practice. This will lead to a low level of variation in practice between practitioners and this low level of variation is evidence of the high-quality care in the standard care model.

By contrast, custom care is designed to deal with clinical problems that are unusual and poorly understood or an unusual response of a well-understood condition to standard interventions. As the healthcare problem (or response) is poorly understood the approach to its solution is iterative rather than sequential. The clinician will act as a problem-solver rather than a rule-follower and will be expected to run through cycles of hypothesis testing and mini-trials and with each iterative cycle there is a reduction in the level of uncertainty. Unlike standard care, the iterative process of custom care involves a high degree of variation. However, custom care can switch to standard care once the iterative process leads to an understanding of the underlying healthcare problem. Also, iterative care may include within it elements of standard care and hence sequential processes as for instance when a certain standard procedure (investigation, trial of treatment, etc.) is carried out on a patient receiving custom care. Psychiatrists and other mental health professionals who have worked with integrated care pathways ${ }^{4}$ that for some years have attempted to standardise good practice will note the similarity with Bohmer's standard care model.

The application of Bohmer's concepts helps to inform a number of important debates. These include having a clearer basis to define the role of the doctor in mental health services, developing a better understanding of the 
nature of clinical autonomy, i.e. when it is appropriate and necessary to behave autonomously and when it is necessary and appropriate to follow rules (guidelines, protocols), and to have a basis for judging when variation becomes an indicator of clinical quality.

\section{How does this relate to the design of mental health services?}

Clearly, doctors including psychiatrists are well suited by their training to custom care. It may be argued that this is also true for a number of other professionals, for example non-medical consultants. Nevertheless, this introduction concentrates on the role of doctors as they form an expensive group of healthcare professionals with a broadly common set of skills. Therefore, any pathways or services that involve dealing with unknown or poorly understood conditions should involve medical practitioners as early as possible during their episode of care. Conversely, standard care that involves the application of sequential processes and requires a high level of rule-following and adherence to protocols can be carried out by non-medical staff. Hence, in principle, once care is standardised in accordance with best available evidence, non-medical staff can become the primary practitioners with little or no direct medical involvement. In mental health services, medical time should be allocated primarily or even exclusively to areas where custom care is required and withdrawn from standard care.

Standardising care in mental health has been successfully implemented in the treatment of depression, for example the IMPACT model $^{5}$ and computerised cognitivebehavioural therapy, which has been approved by the National Institute for Health and Clinical Excellence guidance for depression ${ }^{6}$ and there are many other examples.

Medical time should, therefore, be invested in two main areas: being involved in the direct provision of custom care and having a significant role, together with other professionals, in formulating and updating the sequential processes of standard care. The latter will involve a good deal of hands-on involvement by doctors in service design and in the review of care pathways, standards of care and measures of quality.

The role of the psychiatrist as a prescriber will have relevance to both standard and custom care pathways. In standard care the psychiatrist will have a supervisory role for nurse prescribers, advisory role to general practitioners, leading role in drawing up the standard drug treatment protocols and standards of care, and in some instances direct clinical review of selected patients. In custom care, the psychiatrist will be directly involved as the primary prescriber. Psychiatric trainees will be expected to be primarily involved in standard care during their core training and to be introduced into the delivery of custom care during their specialist training.

\section{Conclusions}

It is possible that mental health problems present a greater degree of complexity and variability within standard care than is the case in other fields of healthcare and this may present a challenge to the utility of this conceptual framework. It is, therefore, expected that vigorous and healthy debate and disagreement will occur regarding where the boundary between standard and custom care lies. I would argue that Bohmer's ideas will help bring this debate out in the open and to make the position of all parties explicit. It will also require that arguments are supported by evidence rather than opinion.

This conceptual framework has a number of advantages. These include the following.

- Setting limits on the process of standardisation. This helps to determine areas that should be intensively standardised and areas where it would be harmful to do so. Standardisation can and should be extended only once a problem becomes well understood and a critical body of evidence becomes available for clinical application.

- Determining when clinical autonomy is justified and when it is not.

- Providing a rational basis for a radical break with traditional work patterns of consultant psychiatrists involving a move away from generic clinical activity to more focused, specialist work.

It is proposed that the above principles will help inform decision-making regarding the design of care pathways in mental health services and should help provide a rational basis for the allocation of medical resources.

\section{About the author}

Riadh T. Abed (FRCPsych) is a Consultant Psychiatrist and Medical Director at the Rotherham Doncaster and South Humber Mental Health NHS Foundation Trust, St Catherine's Hospital, Doncaster.

\section{References}

1 Department of Health. New Ways of Working for Everyone - A Best Practice Implementation Guide. Department of Health, 2007.

2 Department of Health. Responsibility and Accountability: Moving on for New Ways of Working to a Creative, Capable Workforce. Department of Health, 2010.

3 Bohmer, RMJ. Designing Care: Aligning the Nature and Management of Healthcare. Harvard Business Press, 2009.

4 NHS Quality Improvement Scotland. Integrated Care Pathways for Mental Health: Bipolar Disorder, Borderline Personality Disorder, Dementia, Depression, Schizophrenia. NHS Quality Improvement Scotland, 2007 (http://www.nhshealthquality.org/nhsqis/files/MENTALHEALTHICP_ STAND_APR07.pdf).

5 Unützer J, Katon W, Callahan CM, Williams JR JW, Hunkeler E, Harpole $L$, et al. Trial in the primary care setting: a randomized controlled collaborative care management of late-life depression. JAMA 2002; 288: 2836-45.

6 National Institute for Health and Clinical Excellence. Depression (CG90) The Treatment and Management of Depression in Adults. NICE, 2009 (http://egap.evidence.nhs.uk/CG90/). 